\title{
The sex-specific transcriptome of the hermaphrodite sparid sharpsnout seabream (Diplodus puntazzo)
}

\author{
Tereza Manousaki ${ }^{1}$, Alexandros Tsakogiannis ${ }^{1}$, Jacques Lagnel ${ }^{1}$, Elena Sarropoulou' ${ }^{1}$, Jenny Z Xiang ${ }^{2}$,
} Nikos Papandroulakis ${ }^{1}$, Constantinos C Mylonas ${ }^{1}$ and Costas S Tsigenopoulos ${ }^{1 *}$

\begin{abstract}
Background: Teleosts are characterized by a remarkable breadth of sexual mechanisms including various forms of hermaphroditism. Sparidae is a fish family exhibiting gonochorism or hermaphroditism even in closely related species. The sparid Diplodus puntazzo (sharpsnout seabream), exhibits rudimentary hermaphroditism characterized by intersexual immature gonads but single-sex mature ones. Apart from the intriguing reproductive biology, it is economically important with a continuously growing aquaculture in the Mediterranean Sea, but limited available genetic resources. Our aim was to characterize the expressed transcriptome of gonads and brains through RNA-Sequencing and explore the properties of genes that exhibit sex-biased expression profiles.

Results: Through RNA-Sequencing we obtained an assembled transcriptome of 82,331 loci. The expression analysis uncovered remarkable differences between male and female gonads, while male and female brains were almost identical. Focused search for known targets of sex determination and differentiation in vertebrates built the sex-specific expression profile of sharpsnout seabream. Finally, a thorough genetic marker discovery pipeline led to the retrieval of 85,189 SNPs and 29,076 microsatellites enriching the available genetic markers for this species.

Conclusions: We obtained a nearly complete source of transcriptomic sequence as well as marker information for sharpsnout seabream, laying the ground for understanding the complex process of sex differentiation of this economically valuable species. The genes involved include known candidates from other vertebrate species, suggesting a conservation of the toolkit between gonochorists and hermaphrodites.
\end{abstract}

Keywords: Sparidae, Sharpsnout seabream, Diplodus puntazzo, RNA-Seq, Transcriptome, Gonads, Brain, Sex differentiation, Hermaphroditism

\section{Background}

Teleosts exhibit remarkably diverse patterns of sex modes. The way males and females develop, and the molecular mechanisms underlying those differences, vary dramatically among taxa. In teleosts, the decision on an individual's sex ('sex determination') may be due to genetic and/or environmental factors [1-3] with an evident epigenetic component [4]. Apart from the genes that determine sex, downstream genes and pathways drive the development and maintenance of sex-specific phenotypes.

\footnotetext{
* Correspondence: tsigeno@hcmr.gr

'Institute of Marine Biology, Biotechnology and Aquaculture (I.M.B.B.C.),

Hellenic Centre for Marine Research (H.C.M.R.), Heraklion, Greece

Full list of author information is available at the end of the article
}

Those processes define sex differentiation. The genes involved in sex determination and differentiation form the necessary toolkit leading to the sex-specific phenotypes. However, the picture becomes more complicated in cases of hermaphroditism, a rather common sexual system among teleosts.

The molecular processes underlying sex have been deeply studied in model vertebrates like human, mouse, chicken and African clawed frog [5-8]. Several studies on fish have unveiled the genes responsible for sex determination in gonochoristic species, such as Dmy in medaka [9,10], Amhr2 in Tiger pufferfish [11], Sdy in Rainbow trout [12] and Amhy in Patagonian pejerrey [13]. Other studies have revealed loci linked to sex in 
various species (e.g. [14] for stickleback; [15] for tilapia; [16] for Atlantic halibut; [17] for turbot; [18] for zebrafish), even in the hermaphrodite Gilthead seabream [19,20]. All these studies illustrate the large diversity in sex determination among teleosts. However, the genes involved in sex differentiation are considered conserved across vertebrates [21,22], even though alternative scenarios have also been suggested [23]. To get an overview of the genetic toolkit deployed for the development and maintenance of the differences between sexes, whole-transcriptome approaches are required [24]. Several transcriptomic analyses have characterized the expression differences that underlie the two sex phenotypes in fish (e.g. [25-30]) and revealed that the main pathways are present in most species, although the role of each gene might change.

Most studies on understanding sex differentiation have been conducted on gonochoristic taxa. Hermaphroditism, however, is common among teleosts and has evolved repeatedly in different lineages [31]. The two sexual systems are sometimes observed even among phylogeneticallyclose species, suggesting that the molecular pathways involved might not differ dramatically. Sparidae is a teleost family with a wide variety of sex mechanisms [32-34]. Sparids exhibit either gonochorism or various forms of hermaphroditism, such as simultaneous, sequential or rudimentary hermaphroditism (simultaneous: presence of both male and female gonads; sequential: an individual develops first as a functional male and then changes to female or vice versa; rudimentary: immature individuals carry both male and female immature gonad types and during maturation one of the two types develops fully, determining the sex). A recent study on the ancestral reconstruction of sexual patterns in sparids revealed both gonochorism and hermaphroditism in almost every group of the family [31]. Therefore, there is a great potential for understanding the molecular mechanisms underlying gonochorism and hermaphroditism within Sparidae $[31,35]$. To date, considerable effort to unravel the genes involved in sex determination, sex differentiation and sex change have been conducted mainly on the protandrous black porgy, Acanthopagrus schlegelii [35-42]. Further knowledge comes from QTL studies on the protandrous Gilthead seabream, Sparus aurata $[19,20]$, but current knowledge concerning other Sparidae species is poor.

The Sharpsnout seabream, Diplodus puntazzo, is a sparid of great importance for the industry of fisheries and aquaculture. This species is also particularly interesting from an evolutionary point of view, as it has one of the most spectacular reproductive systems; it is rudimentary hermaphrodite with some instances of protandry $[43,44]$. Several studies have investigated various aspects of its biology, including reproduction and development (e.g. [44-46]), but the available information concerning the species' genetic content is limited. To date, this is the case for most sparids, except for the two protandrous species of Sparus aurata and Acanthopagrus schlegelii, which account for more than $90 \%$ of the Sparidae sequences available in GenBank. With modern sequencing technologies, this lack of knowledge can be overcome and ultimately allow the comparison of the genetic networks involved in sex determination and differentiation among closely related species that exhibit different reproductive modes.

Here, we sought to identify and understand the molecular toolkit underlying the sex differences of the expressed transcriptome in the rudimentary hermaphrodite sharpsnout seabream. To that end, we employed an RNA-Sequencing (RNA-Seq) approach [47] aiming at capturing the gene content of sharpsnout seabream that exhibits sex-biased expression pattern. We chose to study both gonad and brain tissues. Gonads were selected to get a comprehensive overview of the genes responsible for the divergence of the primary sex-related structure; brain was included to increase the species genic information and to understand how sex affects brain functions, as shown in gonochoristic fishes (see [48] and references therein). Our results revealed major expression differences between male and female gonads, but only minor differences between male and female brains. Most genes involved in primary pathways of sex determination and differentiation in other vertebrates are present also in the sharpsnout seabream; this implies a conservation of pathways between gonochorists and hermaphrodites. Finally, we constructed a dataset of genes and a resource of genetic markers that will assist future genetic research at the species and family level.

\section{Methods}

\section{Sample collection}

Animal care was carried out according to the "Guidelines for the treatment of animals in behavioural research and teaching" [49]. Hatchery produced sharpsnout seabream (from eggs spawned in October 2010) were reared in tanks supplied with flow-through seawater under ambient conditions at the Institute of Marine Biology, Biotechnology and Aquaculture. Fish were fed daily to apparent satiation using a commercial extruded feed (SKRETTING, Norway and IRIDA S.A., Greece). During the reproductive period (October-December) of 2012 (when fish were $2+$ years old), fish were sampled randomly from the population and were euthanized in a mixture of seawater and ice. Selected individuals were examined for sexual maturation, based on the presence of releasable sperm from the males or the presence of vitellogenic oocytes in the females. At that time, sperm could not be collected from any of the males, but histological evaluation indicated the presence of intratesticular spermatozoa. Females were immature, containing only primary oocytes in their ovaries. 
Gonad and brain tissues were sampled from four male and four female individuals (16 samples in total) in a sterile and RNase-free way and fixed immediately in RNAlater (Applied Biosystems, Foster City, CA, USA). Tissues for RNA extraction were stored at $4^{\circ} \mathrm{C}$ overnight and then transferred to $-80^{\circ} \mathrm{C}$ until further processing.

\section{RNA extraction and sequencing}

To avoid biases regarding different expression in different parts of the same organ, we homogenized the whole brain and male gonad samples whereas in the case of female gonads, because of their large size $(1.2 \mathrm{~g}-4.3 \mathrm{~g})$ we excised three different parts of the organ (the posterior, the anterior and the middle tissue) and homogenized it in QIAzol Lysis buffer (QIAGEN ${ }^{\circ}$ ) following supplier's recommendations.

The disruption and homogenization occurred using Tissuelyser II (QIAGEN ${ }^{\circ}$ ) and steal beads of $5 \mathrm{~mm}$ diameter (QIAGEN ${ }^{\circ}$ ). We used Qiagen's miRNeasy extraction kit, as we targeted on extracting both mRNA and miRNA (only mRNA was analyzed in this study). The quantity of the isolated RNA was measured spectrophotometrically with NanoDrop ${ }^{\circ}$ ND-1000 (Thermo Scientific), while its quality was tested on an agarose gel (electrophoresis in $1.5 \% \mathrm{w} / \mathrm{v}$ ) and further on an Agilent Technologies 2100 Bioanalyzer (Agilent Technologies). The majority of the samples had an RNA Integrity Number (RIN) value higher than 8 (Table 1). This was not the case

Table 1 Sequencing summary

\begin{tabular}{cccccc}
\hline Females id & tissue & RIN & Raw reads & \multicolumn{2}{c}{ Filtered paired reads } \\
\cline { 4 - 6 } & & & & Total & Mapped \\
\hline 1 & gonad & N/A & 20961364 & 11297594 & 9831370 \\
1 & brain & 9.3 & 23980450 & 13193988 & 11095910 \\
2 & gonad & N/A & 27194286 & 14697094 & 12745828 \\
2 & brain & 8.7 & 25146494 & 13764286 & 11511286 \\
3 & gonad & 2.5 & 27629298 & 14487792 & 12602744 \\
3 & brain & 8.9 & 31171108 & 16631824 & 13782126 \\
4 & gonad & 2.5 & 36169194 & 18753838 & 16134938 \\
4 & brain & N/A & 27213654 & 14519532 & 12227478 \\
Males id & tissue & RIN & Raw reads & Total & Mapped \\
5 & gonad & 9.4 & 24782894 & 12751168 & 10650100 \\
5 & brain & 9.2 & 23058518 & 12279090 & 10263134 \\
6 & gonad & N/A & 28182238 & 15353332 & 12958232 \\
6 & brain & 9 & 28819914 & 15465380 & 13044458 \\
7 & gonad & 8.8 & 28895952 & 15281070 & 12634914 \\
7 & brain & 8.4 & 17381152 & 9535140 & 8262496 \\
8 & gonad & 9.5 & 28583256 & 15336768 & 12479306 \\
8 & brain & 9.4 & 30818278 & 16496042 & 13791216 \\
Total & - & - & 429988050 & 229843938 & 194015536 \\
\hline
\end{tabular}

for any of the female gonad samples. Further effort to improve RNA extraction did not yield any higher RIN value. Although NanoDrop values were of the expected range (260/280 ratio [1.98-2.07]), the agarose gel and Bioanalyzer revealed the presence of an enormous peak at $100 \mathrm{bp}$ (Additional file 1: Figure S1). However, several teleost female gonads show a similar RNA profile as reported earlier [50]. This high peak corresponds to 5S rRNA and possibly hampers the correct estimation of the $18 \mathrm{~S}$ and $28 \mathrm{~S}$ rRNA peaks. This was consistent in all female gonad extractions and possibly affected the RIN number as the observed pattern deviated greatly from the expected pattern of RNA content.

Finally, all 16 samples were used for library preparation and sequencing as 100 bp paired reads in 1.5 lanes of a HiSeq2500 following the protocols of Illumina Inc. (San Diego, CA) in the Genomics Resources Core Facility of Weill Cornell Medical College.

\section{Read pre-processing}

Read quality was assessed with FastQC [51] and subjected to quality control with FASTX_Toolkit [52]. Adapters were trimmed with fastx_clipper. Quality trimming was conducted with fastq_quality_trimmer (minimum quality 25, minimum read length 50 ). Reads were further quality controlled by excluding those with more than $5 \%$ of low-quality (quality threshold 25) nucleotides using fastq_quality_filter. After filtering, read pairs were reconstructed with a custom Perl script.

\section{Transcriptome assembly and annotation}

For the assembly, we pooled the filtered reads of all samples and implemented three different trials using SOAPDENOVO [53] ( $\mathrm{kmer}=35$, min length 200 nucleotides $)$, Velvet/Oases $[54,55])(\mathrm{kmer}=35$, min length 200 nucleotides) and Trinity [56] (trinityrnaseq_r2013-02-25; default kmer 25, min length 200 nucleotides). The three candidate assemblies were evaluated by BLASTn [57] against Oreochromis niloticus, Oryzias latipes and Gasterosteus aculeatus cDNA dataset downloaded from Ensembl database [58] with an $e$-value threshold of $10^{-9}$. The assembly produced by Trinity had the highest number of significant similarity with unique genes from all three teleost species and was selected.

To assess the assembled transcripts and exclude the spurious ones, we pooled all the reads and mapped them to the selected assembly with Bowtie [59] within RSEM [60] using the script available in trinity utilities run_RSEM_align_n_estimate.pl. Putative transcripts with less than $1 \%$ of a locus reads mapped to that particular isoform (IsoPct $<1$ ) were eliminated (as proposed in [61]). The same was done for those with Fragments Per Kilobase of transcript per Million mapped reads (FPKM) values less than 0.3. The choice of FPKM threshold was 
based on BLASTn similarity searches (e-value threshold $10^{-10}$ ) against $O$. niloticus cDNA sequences. The unfiltered transcripts dataset had significant similarity with 18,527 O. niloticus genes, while the filtered assembly had significant similarity with 17,346 out of 21,462 genes reported in Ensembl. This dataset constituted the final assembled transcriptome given that the selected threshold value resulted in elimination of 255,891 possibly spurious transcripts, while the hits to unique genes of $O$. niloticus were only slightly reduced.

To annotate the assembled transcripts, we conducted a BLASTx similarity search against the NCBI protein database $n r$ (e-value threshold $10^{-9}$; keeping the top ten hits). BLASTx was done in parallel using NOBlast [62]. The output was used in Blast2GO [63] where gene ontology terms were retrieved and assigned to the transcripts (only the longest transcript was used per locus). The open reading frames (ORF) were extracted per sequence with the EMBOSS program getorf. InterProScan [64] was run on the longest ORF per transcript. The run was done in parallel splitting the query in 100 subqueries and merging the output with custom scripts. GO terms derived from InterProScan were merged with GO terms derived from BLASTx against $n r$. In cases where accurate orthology inference was of interest (e.g. for identifying the orthologs of the genes associated with sex in other taxa), we implemented a reciprocal BLASTn hit approach of the annotated sequences of medaka, tilapia and other fish from Ensembl against sharpsnout seabream assembled transcripts. When the gene of interest had significant similarity with the assembled transcripts, we used the top hit in a BLASTn search towards the transcriptome of the starting species. If that step returned the initial transcript, we assumed orthology between the two.

\section{Differential expression}

The paired reads of each sample were mapped to the assembly with Bowtie and abundance was estimated with RSEM v. 1.2.4, as implemented in the trinity script run_RSEM_align_n_estimate.pl. The estimated expected counts for each sample, at the gene level, were extracted and used for the analysis of differential expression conducted in DESeq [65], a software considered accurate and conservative for differential expression analyses $[66,67]$. Samples were grouped according to sex and expression was compared for each tissue separately, following the developers' manual (FDR threshold of 0.05). Due to a reported difficulty in assessing differential expression when a gene is expressed only in one group [66], we considered as significant those genes only when the expression in the other group was higher than 30 normalized counts in total.

\section{Detection of genetic markers}

The assembled sequences were scanned for microsatellites with Phobos [68]. We detected non-exact Short Tandem Repeats (STRs) with 2-10 repeat unit length and a minimum length of 20 nucleotides. A custom Perl script was used to parse the output.

Single Nucleotide Polymorphisms (SNPs) were detected with SAMTools [69] and VCFtools [70]. Alignment (.bam) files produced through mapping for gonads and brain were merged for each individual. Alignment files were further filtered for mapping quality (threshold 75), number of mismatches to the reference ('NM' threshold 10) and for proper pair mapping with BamTools [71]. Then, they were sorted and piled (with SAMTools function 'mpileup') to detect candidate SNPs. Custom Perl scripts were used to eliminate SNPs that formed clusters ( $>1$ variant every 4 bases) to exclude hypervariable regions where accurate alignment is difficult. Those with SNP QUAL $<25$, total high quality bases coverage below 15 and minor allele read ratio $<0.2$ were excluded [72]. In each locus we kept only the SNPs belonging to the transcript with the highest SNP number. Finally, SNPs were categorized to synonymous, non-synonymous and those that belong to the transcripts UTRs. For that, we first retrieved the predicted ORF per transcript with the highest similarity to tilapia proteins ( $e$-value threshold $\left.10^{-10}\right)$. Then, we evaluated whether each SNP is located within the coding region defined by the ORF, and for those located within the coding region if it causes a synonymous or a nonsynonymous mutation using a Perl script.

To identify SNPs for which genotyping can be conducted robustly we applied extra filtering steps. Those included the genotype quality ('GQ') score $>=20$ as provided by SAMtools and VCFtools pipeline and required minimum coverage of the SNP site per sample $>5$ for all samples. Note that the genotype quality is a function of the probability that the genotype call is wrong given that the site is variable. SNPs that passed the extra criteria were used for a PCA and a relatedness analysis with the $\mathrm{R}$ package SNPRelate [73]. For the Relatedness analysis, we estimated Identity By Descent with the Maximum Likelihood method and calculated the kinship coefficient. Finally, all scripts used in the study are available upon request.

\section{Results}

\section{Sharpsnout seabream assembled transcriptome}

Illumina HiSeq2500 sequencing yielded 429,988,050 paired reads $(214,994,025$ read pairs) (Table 1$)$. The filtering process resulted in 229,843,938 paired and 74,027,237 single or orphan high quality reads used for the transcriptome assembly.

The initial assembly process produced 374,149 putative transcripts (N50: 1,712 nucleotides, mean length: 
871 nucleotides). Following further editing and transcript quality assessment (see Methods), we limited our dataset to 118,258 transcripts belonging to 82,331 loci (N50: 2,092; mean length: 1,352 nucleotides) (Figure 1). The unfiltered transcripts dataset had significant similarity to 18,527 O. niloticus genes, while the filtered assembly had significant similarity to 17,346 out of 21,462 genes reported in Ensembl. Given that the filtering resulted in elimination of 255,891 possibly spurious transcripts, while the hits to unique genes of $O$. niloticus were only slightly reduced, the restricted dataset constituted the final transcriptome. Out of the 82,331 loci, 31,895 had significant BLASTx similarity hits with publicly available protein sequences, 22,574 of the loci were assigned GO terms and 40,823 had an InterProScan protein domain match. The great majority of the top-hits of the assembled transcripts had significant similarity with sequences of Maylandia zebra, Oreochromis niloticus, Takifugu rubripes, Oryzias latipes and Dicentrarchus labrax. Further, 14,041 of the genes without any significant similarity to known proteins had InterProScan protein domains assigned.

\section{Comparative expression profiling between males and females}

The global gene expression pattern observed in the tissues of the eight individuals used in this study is summarized in a principal component analysis (PCA) (Figure 2). The picture obtained shows a clear separation between female and male gonads with remarkable variation within the two groups. On the contrary, brain expression patterns are similar between males and females in this general overview.

A separate PCA of the brain global expression values showed a scattered pattern with some overlap between the groups of males and females (Figure 2). Notably, one of the male brain samples had an extreme profile compared to the other male or female samples. To test whether this was due to library construction error, we mapped the reads to Sparus aurata ribosomal RNA ( $r$ RNA) to find no relation of $r$ RNA content to the outlier (for the majority of samples $0-0.05 \%$ of the reads were mapped on $r$ RNA). Thus, this sample was excluded from the differential expression analysis. For gonads, global expression patterns showed once again a clear separation of males and females (Figure 2). Sex-specific expression patterns were evaluated separately for brain and gonad tissues and are described below.

\section{Male vs. female gonads expression patterns}

In gonads, male and female expression patterns differed greatly. Out of the total 82,331 loci, 71,387 had an estimated abundance of at least one pair of reads in gonad samples. The differential expression analysis revealed 5,558 loci up-regulated in female and 8,072 loci up-regulated in male gonads (Figure 1; Additional file 2: Figure S2; Additional file 3: Table S1). Clustering of the samples based on the top 40 differentially expressed genes grouped males and females separately (Figure 3).

The putative functions of those genes include development, signal transduction and metabolism (Figure 4). To test whether certain functions are more frequently observed in one of the two sexes, we conducted a Fisher's exact test in Blast2GO comparing the GO terms of the male versus the female over-expressed genes (each gene was represented by the longest transcript; $p$-value 0.05; Table 2; see Additional file 4: Table S2 for complete list). The main GO terms associated with the genes
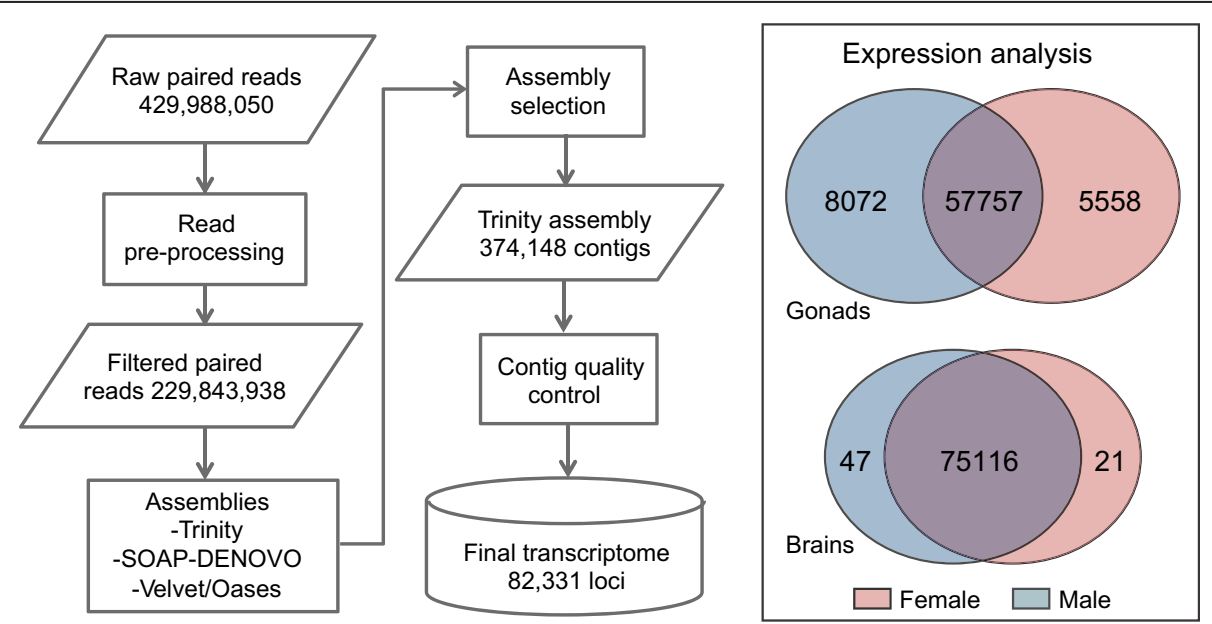

Figure 1 The pipeline followed to build the assembly and the male versus female expression profiles. On the left, flow chart of the steps implemented from raw reads to the selection of the final assembled loci. On the right, Venn diagram showing the loci commonly and differentially expressed in the two sexes for brains and gonads. 

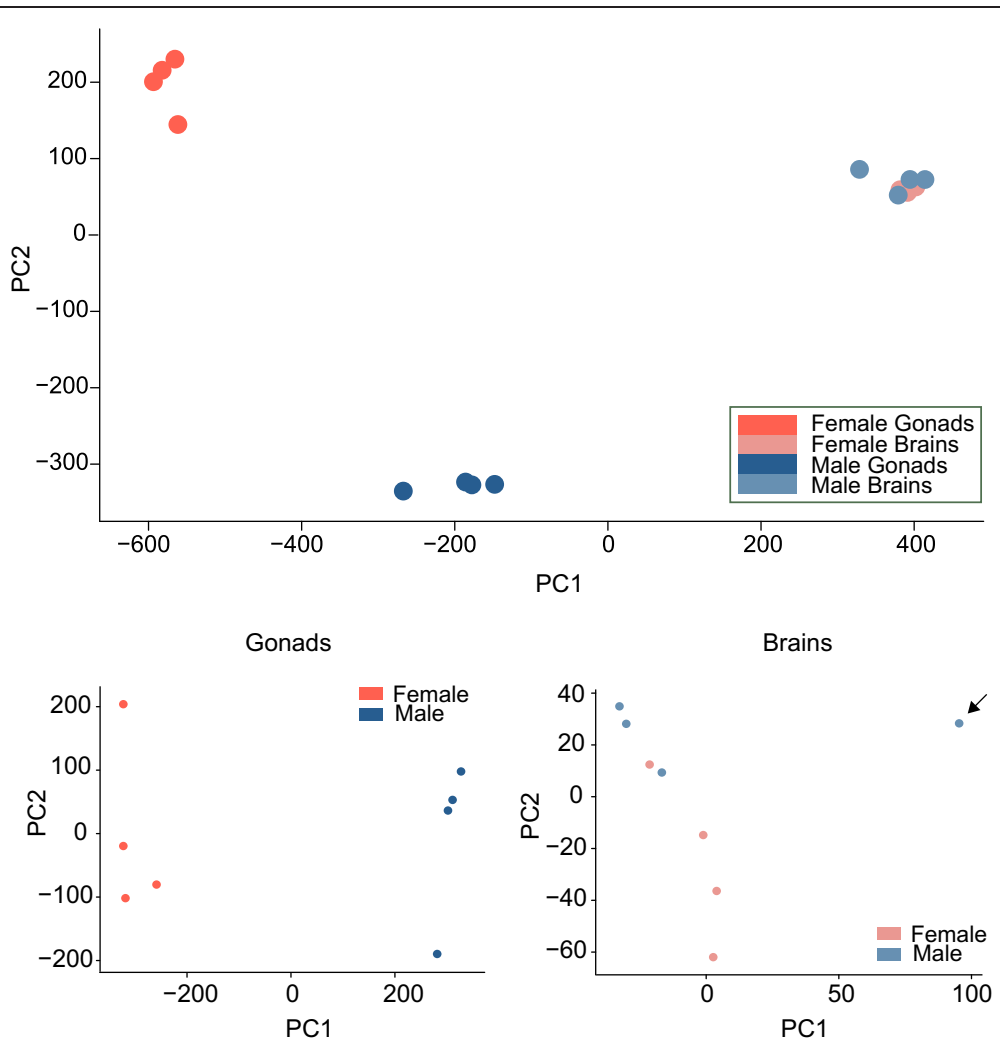

Figure 2 Principal component analysis of the expression profiles. On top, the global expression profiles of gonad and brain samples of male and female individuals analyzed in a PCA. At the bottom, brain and gonad samples are analyzed separately. The brain sample pointed by the arrow is considered an outlier and was excluded from the differential expression analyses.

over-expressed in females are involved in metabolism, while in males in signaling and regulation of transcription.

\section{Sex determination and differentiation genes in gonads}

The expression profiles of genes known to be involved in sex determination and differentiation (as reviewed in
[28]) were closely examined (Table 3). Orthology with other genes was assessed with the reciprocal best hit approach (See Methods). Out of 44 target genes, 42 are found within the assembled transcriptome. The only genes not found were Fgf9 and Dmrt6. Dmr6 is absent from the teleost lineage according to Ensembl orthology
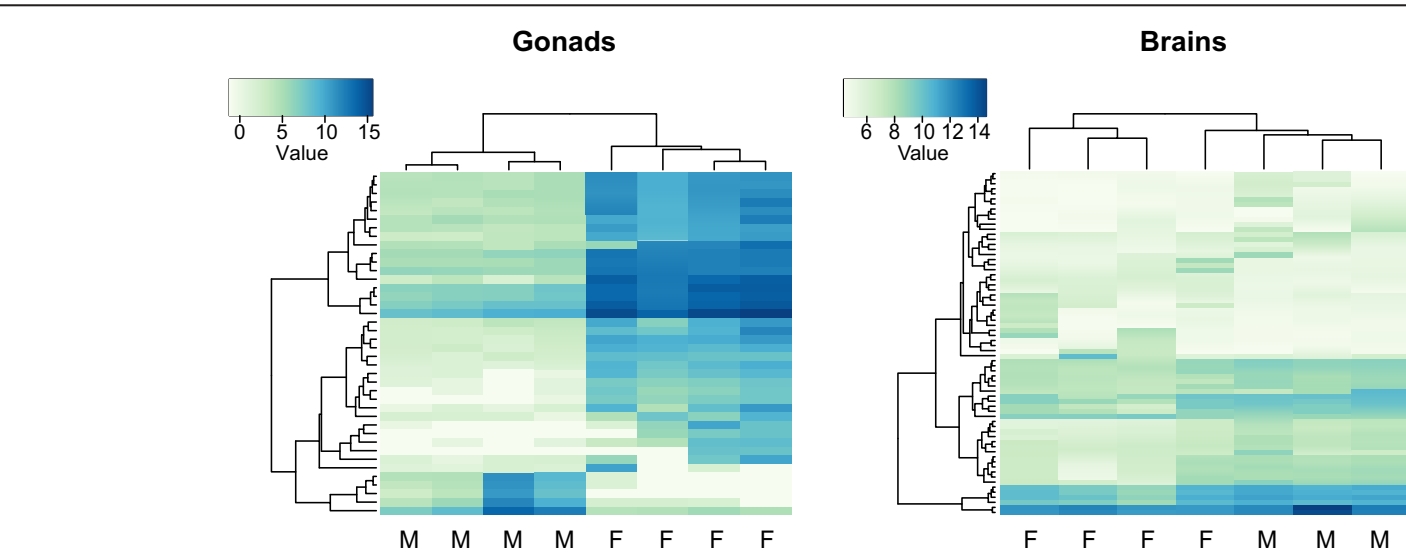

Figure 3 Heatmap of the differentially expressed genes for gonad and brain samples. Heatmap of the variance-stabilized transformed count data of gonad and brain samples for the differentially expressed genes between male and female individuals. Note that only the top 40 genes are included in the gonad heatmap; for the brain heatmap all genes are included. 


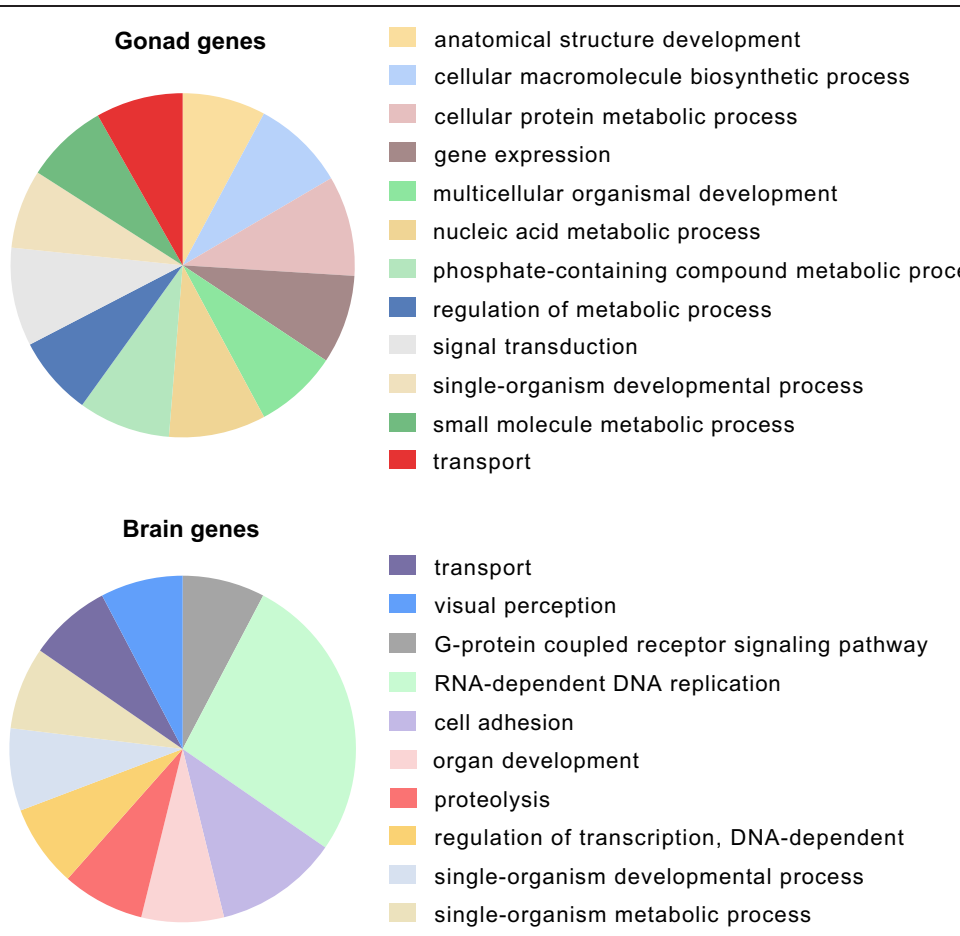

Figure 4 Gene Ontology profiling of gonad and brain samples. Pie charts representing the GO terms (Biological Process) associated with the genes over-expressed in gonad and brain samples. For gonads only terms with $>700$ sequences are shown.

inference and Fgf9 might be not expressed, not sequenced or lost from sharpsnout seabream. Sixteen genes are significantly differentially expressed between the two gonad types, while 15 change in the 'expected' direction of expression, assuming that the expression direction observed in other vertebrates is conserved in sharpsnout seabream. Only Dhh shows an overexpression in female gonads, opposite to the expected.

Further data mining revealed eight loci containing the GO term "sex differentiation" or "sex determination", and 34 more related to the terms "female" or "male" (e.g. female/male gonad development, female/male meiosis,

Table 2 Comparison of male versus female up-regulated gene functions

\begin{tabular}{|c|c|c|c|c|c|c|}
\hline GO-ID & GO-Term & FDR & P-Value & Females seq. count & Males seq. count & Over-represented \\
\hline GO:0006412 & translation & 7.8537E-48 & $9.13693 \mathrm{E}-51$ & 186 & 6 & females \\
\hline GO:0008152 & metabolic process & $3.14977 \mathrm{E}-46$ & 4.07157E-49 & 1707 & 1184 & females \\
\hline GO:0022613 & ribonucleoprotein complex biogenesis & $3.00773 \mathrm{E}-35$ & 7.77593E-38 & 123 & 1 & females \\
\hline GO:0071704 & organic substance metabolic process & $2.96565 \mathrm{E}-34$ & $8.05049 \mathrm{E}-37$ & 1497 & 1058 & females \\
\hline GO:0044237 & cellular metabolic process & $5.57895 \mathrm{E}-34$ & 1.58657E-36 & 1392 & 959 & females \\
\hline GO:0010467 & gene expression & $7.71755 E-34$ & $2.29451 \mathrm{E}-36$ & 549 & 240 & females \\
\hline GO:0042254 & ribosome biogenesis & $1.02323 \mathrm{E}-31$ & $3.3067 E-34$ & 106 & 0 & females \\
\hline GO:0044238 & primary metabolic process & $1.6642 \mathrm{E}-31$ & 5.80837E-34 & 1445 & 1028 & females \\
\hline GO:0006396 & RNA processing & $3.75931 \mathrm{E}-31$ & 1.40926E-33 & 146 & 11 & females \\
\hline GO:0043170 & macromolecule metabolic process & $1.84976 \mathrm{E}-26$ & $9.08619 E-29$ & 1119 & 758 & females \\
\hline GO:0007154 & cell communication & $2.14665 \mathrm{E}-29$ & $8.87965 \mathrm{E}-32$ & 289 & 641 & males \\
\hline GO:0044700 & single organism signaling & $2.48667 \mathrm{E}-29$ & 1.0929E-31 & 280 & 628 & males \\
\hline GO:0023052 & signaling & $2.48667 \mathrm{E}-29$ & $1.0929 \mathrm{E}-31$ & 280 & 628 & males \\
\hline GO:0007165 & signal transduction & $1.98929 \mathrm{E}-28$ & 9.00013E-31 & 269 & 606 & males \\
\hline
\end{tabular}

Fisher's exact test showing top GO terms significantly over-represented in loci up-regulated in male or female gonad samples. For a complete list see Additional file 4: Table S2. 
Table 3 Sharpsnout seabream genes involved in sex determination and differentiation in other vertebrate taxa

\begin{tabular}{|c|c|c|c|c|c|c|}
\hline Starting sequence & Species & $\begin{array}{l}\text { Gene } \\
\text { name }\end{array}$ & Gene description & Locus & $\begin{array}{l}\text { Exp. up- } \\
\text { regulation }\end{array}$ & $\begin{array}{l}\text { Obs. up- } \\
\text { regulation }\end{array}$ \\
\hline ENSORLT00000015555 & medaka & Wt1 & Wilm's tumour suppressor-1 & comp117315_c1 & $M / F$ & M \\
\hline ENSORLT00000012371 & medaka & Wt1b & Wilm's tumour suppressor-1 & comp117315_c1 & $M / F$ & M \\
\hline ENSORLT00000014817 & medaka & Dax1 & $\begin{array}{l}\text { dosage-sensitive sex-reversal-adrenal hypoplasia } \\
\text { congenital-critical region of } X \text { chromosome, gene } 1\end{array}$ & comp87968_c1 & M & - \\
\hline ENSXMAT00000015647 & platyfish & Dmrt1 & double sex and mab-3 related transcription factor 1 & comp110196_c1 & M & M \\
\hline ENSONIT00000017862 & tilapia & Dmrt3 & double sex and mab-3 related transcription factor 3 & comp88038_c1 & M & M \\
\hline ENSLOCT00000007854 & spotted gar & Dmrt6/Dmrtb1 & double sex and mab-3 related transcription factor 6 & - & M & - \\
\hline ENSORLT00000021733 & medaka & Gata-4 & GATA-binding protein 4 & comp114090_c1 & $M / F$ & M \\
\hline ENSORLT00000025798 & medaka & Fgfg & fibroblast growth factor 9 & - & M & - \\
\hline ENSORLT00000012578 & medaka & Fgf20 & fibroblast growth factor 20 & comp85925_c1 & M & - \\
\hline ENSONIT00000015290 & tilapia & Dhh & desert hedgehog & comp116302_c0 & M & $\mathrm{F}$ \\
\hline ENSONIT00000006022 & tilapia & Amh & anti-Mullerian hormone & comp107049_c0 & M & M \\
\hline ENSONIT00000015711 & tilapia & Amhr2 & anti-Mullerian hormone receptor, type ॥ & comp111277_c1 & M & - \\
\hline ENSONIT00000022132 & tilapia & $\operatorname{Ar}$ & androgen receptor & comp108409_c2 & M & M \\
\hline ENSONIT00000016191 & tilapia & Arb & androgen receptor beta & comp122243_c0 & M & - \\
\hline ENSONIT00000025518 & tilapia & Wnt4 & Wingless-type MMTV integration site family 4 & comp94275_c1 & $\mathrm{F}$ & - \\
\hline ENSONIT00000016320 & tilapia & Wnt4b & Wingless-type MMTV integration site family $4 \mathrm{~b}$ & comp308556_c0 & $\mathrm{F}$ & - \\
\hline ENSONIT00000020541 & tilapia & Rspo-1 & R-spondin-1 & comp102190_c3 & $\mathrm{F}$ & - \\
\hline ENSONIT00000009116 & tilapia & Ctnnb1 & catenin $\beta-1$ & comp112822_c5 & $\mathrm{F}$ & $\mathrm{F}$ \\
\hline ENSONIT00000026114 & tilapia & Fox 12 & forkhead box transcription factor $L 2$ & comp95964_c0 & $\mathrm{F}$ & - \\
\hline ENSONIT00000017958 & tilapia & Fst & follistatin & comp112642_c0 & $\mathrm{F}$ & - \\
\hline ENSONIT00000000198 & tilapia & Cyp19a1 & aromatase a & comp117077_c0 & $\mathrm{F}$ & $\mathrm{F}$ \\
\hline ENSONIT00000008152 & tilapia & Cyp11b & 11 beta-hydroxylase & comp108630_c0 & M & M \\
\hline ENSORLT00000018193 & medaka & Esrr & oestrogen receptor 1 & comp117911_c10 & $\mathrm{F}$ & - \\
\hline ENSORLT00000013323 & medaka & Era & oestrogen receptor a & comp112646_c1 & $\mathrm{F}$ & - \\
\hline ENSORLT00000022182 & medaka & $\operatorname{Er} \beta$ & oestrogen receptor $\beta$ & comp112065_c1 & $\mathrm{F}$ & - \\
\hline ENSORLT00000022555 & medaka & $\operatorname{Er} \beta 2$ & oestrogen receptor $\beta 2$ & comp108758_c0 & $\mathrm{F}$ & - \\
\hline ENSORLT00000009986 & medaka & Sox9b & SRY-related HMG-box 9 & comp113806_c6 & M & - \\
\hline ENSORLT00000023249 & medaka & $50 \times 9$ & SRY-related HMG-box 9 & comp108517_c9 & M & - \\
\hline ENSONIT00000006616 & tilapia & $50 \times 8$ & SRY-related HMG-box 8 & comp113806_c6 & M & - \\
\hline ENSONIT00000024737 & tilapia & Sox8a & SRY-related HMG-box 8 & comp70271_c1 & M & - \\
\hline ENSONIT00000010558 & tilapia & Sox10 & SRY-related HMG-box 10 & comp116475_c6 & M & - \\
\hline ENSONIT00000022743 & tilapia & Sox10(2 of 2) & SRY-related HMG-box 10 & comp114808_c13 & M & - \\
\hline ENSONIT00000009624 & tilapia & Gsdf & Gonadal soma derived factor & comp113923_c0 & M & - \\
\hline ENSONIT00000004791 & tilapia & Pdgfaa & platelet-derived growth factor alpha a & comp113241_c2 & M & M \\
\hline ENSONIT00000006486 & tilapia & Pdgfab & platelet-derived growth factor alpha b & comp107178_c1 & M & - \\
\hline ENSONIT00000016484 & tilapia & Pdgfba & platelet-derived growth factor beta a & comp117159_c4 & M & - \\
\hline ENSONIT00000010783 & tilapia & $P d g f b b$ & platelet-derived growth factor beta $\mathrm{b}$ & comp113875_c0 & M & M \\
\hline ENSONIT00000016453 & tilapia & Pdgfrb2 & platelet-derived growth factor receptor, beta & comp104719_c1 & M & M \\
\hline ENSONIT00000022434 & tilapia & Pdgfrb1 & platelet-derived growth factor receptor, beta & comp113376_c0 & M & - \\
\hline ENSONIT00000003760 & tilapia & Pdgfra & platelet-derived growth factor receptor, alpha & comp116299_c0 & M & M \\
\hline ENSONIT00000025493 & tilapia & Sf1 & steroidogenic factor-1 & comp120045_c3 & $M / F$ & M \\
\hline
\end{tabular}


Table 3 Sharpsnout seabream genes involved in sex determination and differentiation in other vertebrate taxa (Continued)

\begin{tabular}{|c|c|c|c|c|c|c|}
\hline ENSONIT00000009791 & tilapia & Srd5a1 & steroid-5-alpha-reductase, alpha polypeptide 1 & comp121181_c8 & $M$ & - \\
\hline ENSONIT00000008643 & tilapia & $\operatorname{srd} 5 a 2$ & 5a-reductase 2 & comp106131_c0 & M & - \\
\hline ENSORLT00000000749 & medaka & Srd5a3 & 5a-reductase 3 & comp115080_c0 & $M$ & - \\
\hline
\end{tabular}

The expected direction of expression (Exp. Up-regulation) is estimated from previous reports on other vertebrates, while the observed (Obs. Up-regulation) is the direction found in sharpsnout seabream gonads. M: over-expression observed in males, F: over-expression observed in females.

female pregnancy, etc.). From those 42 genes in total, 17 were significantly differentially expressed in the gonads (Additional file 5: Table S3) including Dmrt1 and Ctnnb1.

To search for potential genes that are present or absent from the two gonad tissues, we listed those differentially expressed loci that had an estimated abundance of absolutely zero counts in one gonad type, but at least a minimum of expression in the other (mean count $>30$ ). We found 95 loci fulfilling those criteria (Additional file 6: Table S4). The majority (89 out of 95) exhibited a malebiased expression. Those loci were used in a similarity search (BLASTp) against tilapia proteins ( $e$-value threshold $\left.10^{-10}\right)$. Our search retrieved 21 tilapia genes as top hits (Additional file 6: Table S4). Through Ensembl BioMART interface, we retrieved the scaffolds those genes are located in tilapia and downloaded the orthologous genes for stickleback and human. This comparison showed that some of the tilapia genes are located in the same scaffold. Search in stickleback showed that some of the genes located in different scaffolds in tilapia are found in the same chromosome in stickleback. Finally, the human ortholog of the genes Fam70A and Xpnpep 2 are both located on the $\mathrm{X}$ chromosome (Additional file 6: Table S4).

\section{Male vs. female brain expression patterns}

In brain, 75,184 loci had an estimated abundance of more than one pair of reads. Male and female overall expression patterns were indistinguishable (Figure 2). However, the comparison of brain expression patterns (after excluding the outlier sample) between males and females revealed 68 genes (Figure 1; Additional file 3: Table S1) with significant expression difference between the two sexes (21 were over-expressed in females and 47 in males). Clustering of the samples based on all differentially expressed genes led to the grouping of one female with the males as observed also in the PCA conducted in total expression profiles (Figure 3; Figure 2).

The main GO terms associated with the genes found in brain comparison are related to transposable elements, developmental processes, visual perception and signaling among others (Figure 4). Fisher's exact test did not reveal any significantly over-represented term in the brain dataset compared to the whole assembly, probably due to the small size of the dataset.

\section{Genetic marker discovery}

We searched for two types of markers, SNPs and STRs. For the SNP discovery, we combined the reads obtained from the brain and gonad of each individual to increase the individual coverage and then applied various quality filters (see Methods, Table 4). In total, we found 85,189 SNPs, that pass the quality criteria, located in 30,291 loci (Additional file 7: Table S5). From those loci, 9,997 are significantly similar to known tilapia cDNA sequences and contain at least one predicted ORF. From the total SNPs within those 9,997 loci (30,278 SNPs), 10\% were in the first, $\sim 7 \%$ in the second and $\sim 41 \%$ in the third codon position. The rest were in the non-coding regions (5' and 3' UTRs) (Figure 5).

From the loci containing SNPs, $\sim 25 \%$ were significantly differentiated in males and females (37 in brain and 7,592 in gonads). Especially for the gonads, in which the total number of differentially expressed genes was 13,630 , this is way more than expected by chance (twosample $z$ test on proportions; $p$-value $<0.05$ ).

The required coverage and quality criteria applied secure a robust genetic marker discovery. In an attempt to genotype all individuals in certain loci, we applied further criteria requiring certain depth and quality per individual SNP calling (see Methods). Those filters reduced dramatically the number of SNPs that are exploitable for genotyping to 1,009 SNPs (Additional file 8: Table S6). The individual genotypes for all these SNPs were used in a PCA (Additional file 9: Figure S3) and a relatedness analysis (Additional file 10: Table S7). The average kinship coefficient (probability that two alleles randomly chosen from two individuals are Identical By Descent)

Table 4 SNP discovery filters

\begin{tabular}{ccc}
\hline & Filtering summary & \\
\hline Criteria & SNPs & Loci \\
\hline Overall & 354323 & 79898 \\
Delete clusters & 308353 & 78721 \\
Quality $>$ 25 & 244875 & 53911 \\
Coverage $>$ 15 & 149232 & 36829 \\
Read allele ratio $>$ 0.2 & 96942 & 30291 \\
1 transcript per locus & 85189 & 30291 \\
\hline
\end{tabular}

In each step the remaining SNPs and the corresponding loci are shown. 


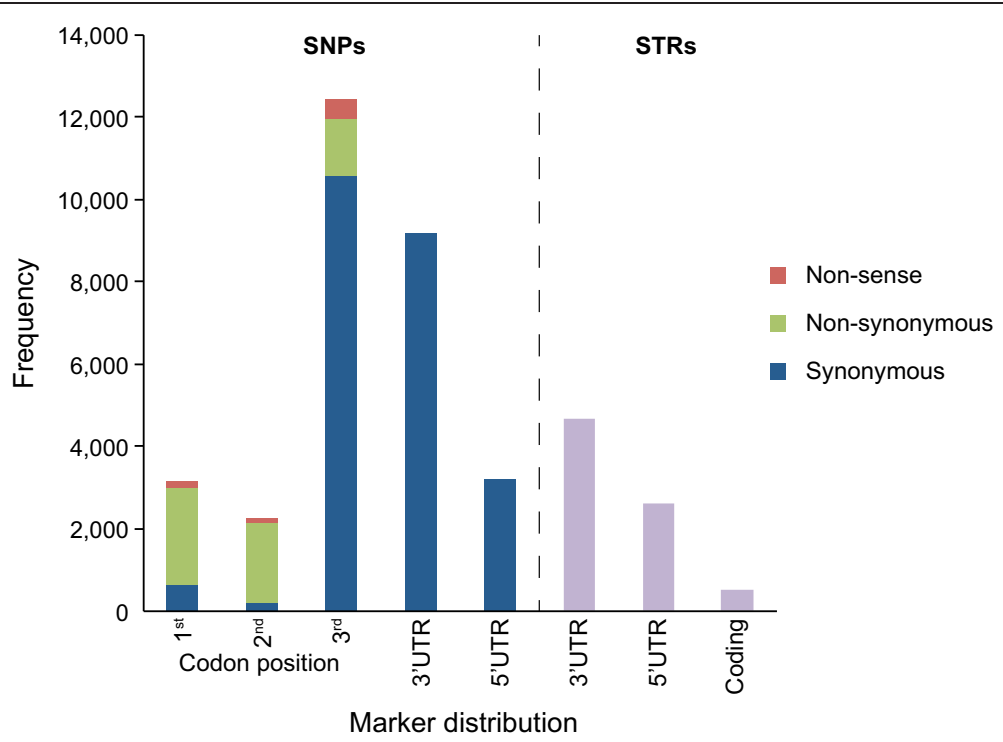

Figure 5 Genetic markers distribution at the coding loci. SNPS and STRs within coding regions are categorized according to their distribution at the codon position (for SNPs only) or 5' and $3^{\prime}$ UTRs.

between pairs is $\sim 0.10$ (min: 0.04 ; max: 0.18). No parent-offspring relationship is observed (expected kinship coefficient 0.25 ). Note that linkage disequilibrium-based SNP pruning was not conducted due to lack of information regarding linkage of SNPs.

Our search for STRs revealed 29,076 candidates located in 16,377 loci (23,759 transcripts) (Additional file 7: Table S5). From the discovered microsatellites, 12,625 STRs $(\sim 43 \%)$ are found in genes that code for proteins based on similarity searches with tilapia cDNA and 7,804 had a predicted ORF $>60$ aa. The latter were used to study the STR distribution in the 5'UTR, 3'UTR and coding regions (Figure 5). The unit-length distribution of the discovered STRs is shown in Figure 6. Finally, STRs were found in 18 genes differentially expressed in brain and 3,713 in gonads.

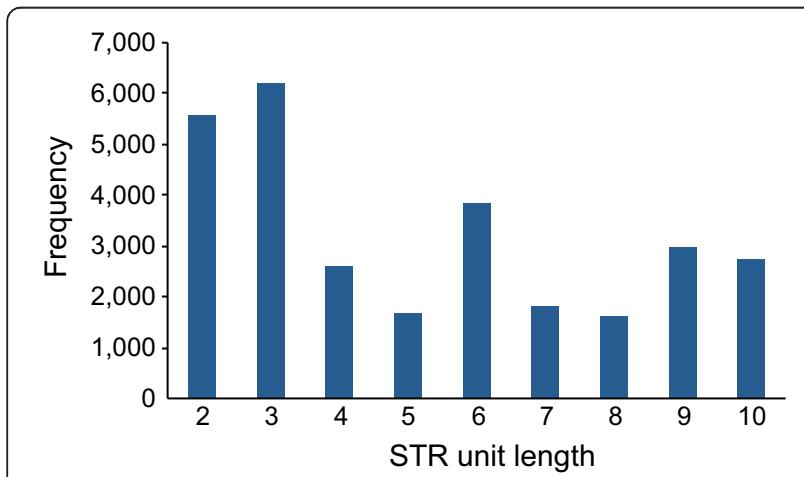

Figure 6 Unit length distribution of identified STRs. Plot of STRS frequency based on unit length.

\section{Discussion}

The analyses presented explore the transcriptomic landscape of female and male gonad and brain tissues providing the first assessment of the molecular toolkit underlying the reproductive biology of a rudimentary hermaphrodite fish. We investigated the expression differences observed between sexes and tracked the expression of known targets of sex determination and differentiation. Finally, we provided a catalogue of SNPs and STRs that will assist following genetic research of the economically important sharpsnout seabream.

\section{The gonad and brain transcriptome of sharpsnout seabream}

Gonad and brain expression profiling was conducted on males and females, with four individuals/biological replicates in each of the two groups. The reason we chose relatively immature individuals was two-fold. First, we sought to capture the transcriptomic differences between the two sexes in a stage where the two gonad types are clearly formed and sex is easily identifiable in this rudimentary hermaphrodite species. Second, we aimed at genes that contribute to sex differentiation, but upstream players that might play a role in sex determination as well. The stage selection was appropriate for characterizing the sexrelated transcriptomic profiles of sharpsnout seabream, since we identified the great majority of previously reported sex differentiation and determination genes of various taxa in our assembled sequences.

The general pattern observed in the expression analyses revealed a homogeneous global expression among brain samples for both sexes (Figure 2). On the contrary, 
gonads diverge greatly among individuals. Although, the two sexes clearly form two groups, there is great withingroup variation. This might reflect the actual developmental stage of each individual. All individuals used in the study are of the same stage, but we cannot rule out the possibility that within the observed developmental stages different biological and molecular processes take place through a series of finer phases exhibiting alternative expression profiles.

\section{Male vs. female gonads expression patterns}

The most striking aspect in the expression profiles of male and female gonads is the magnitude of the discovered differentially expressed genes $(\sim 20 \%$ of the total 71,387 loci expressed in gonads). A similar pattern is obtained from the PCA where the distances between the two gonad types are comparable to that between any of the two gonad types and the brain (Figure 2). This probably reflects their functional divergence. It is noteworthy that many more genes are over-expressed in male rather than female gonads ( $\sim 60 \%$ of the gonad DE genes). This male-bias has been observed in other fish species gonad comparisons (zebrafish: [25,26]; tilapia: [30]) or in whole organism male-female comparison (platyfish: [27]). However, given the lack of knowledge on the genetic and/or environmental mechanisms of sex determination and differentiation in sharpsnout seabream (or any other sparid), we cannot infer whether this is linked to the genetic architecture of the species or reflects other biological phenomena. The presence of genes expressed in the gonads of only one of the two sexes may be linked to the genetic architecture of sharpsnout seabream. The syntenic relationship of several of those genes observed in other species suggests a possible syntenic relationship in sharpsnout seabream and a common regulatory mechanism driven by the physical proximity on the genome. This can be tested in the future, e.g. with the construction of a genetic linkage map.

Multiple previous studies on vertebrates provide us with numerous candidate genes with possible involvement in sex determination and differentiation. A detailed search for those genes in the assembled transcriptome of sharpsnout seabream revealed that almost all are present, and some are differentially expressed in the gonads (Table 3). To start with the male phenotype, Dmrt1 is the gene with the most prominent role in sex determination and spermatogenesis across vertebrates $[74,75]$. In teleosts, it is highly linked with gonad development and maintenance (see [76] for a review). In Sparidae, Dmrt1 has been implicated in the fate of the ovotestis in the protandrous black porgy Acanthopagrus schlegelii, and eliminating it results in a change from male to female [35,77]. In our data, Dmrt1 is up-regulated in male gonads implying a strong role in the development and maintenance of the male phenotype in the hermaphrodite sharpsnout seabream. Amh, and its receptor Amhr2, are both main candidates for triggering and maintaining the male phenotype. In our data, Amh is over-expressed in male gonads suggesting an important role in sharpsnout seabream. On the contrary, Amhr2 exhibits similar expression levels in both gonad types. Both Amh and Amhr2 have been linked to gonadal development in the protandrous black porgy [78]. Focused research on sharpsnout seabream and other Sparidae will reveal their specific role. Amh is tightly linked to Sox 9 and Sf1 [79]. The male factor known to act on Sertoli cells, Sox9, was not differentially expressed in the sharpsnout seabream gonads. Other studies have showed that it is upregulated in male Siberian sturgeons with undifferentiating gonads - in contrast to mature ones - [80], and it is overexpressed in the male immature gonads of sablefish [81]. Thus, Sox 9 might play a role in gonadal development at stages earlier than those studied here (e.g. see [82]). In contrast to $\operatorname{Sox} 9, \operatorname{Sf1}$ has significantly higher expression in male gonads. This factor might be tightly linked to the observed up-regulation of $A m h$. Male phenotype is reinforced by the high expression of androgen receptor, while Dax1- another gene involved in testis development- is similarly expressed between male and female gonads. The same pattern has been observed in tilapia [83], seabass [84] and medaka [85] where Dax1 showed no expression difference suggesting a role in both gonad types. Interestingly, $D h h$, one of the genes involved in testis differentiation and development in humans and mice [86,87], is linked with the function of female rather than male gonads in sharpsnout seabream. However, in mammals it is expressed in both testes and ovaries [88-90]. Finally, multiple other genes strongly related with vertebrate male gonads are found within the sharpsnout seabream transcriptome (Additional file 3: Table S1).

Concerning the genes involved in female gonads, we observed significant over-expression in two key players of ovarian development, the ovarian aromatase (Cyp19a1a) and $\beta$-catenin (Ctnnb). Cyp19a1 is a central component of ovarian steroidogenesis (converts androgens to estrogens). It is a conserved protein with a strong role in the ovarian development of vertebrates (see [91]). In fish, it is important not only for gonochoristic [92-95], but also hermaphrodite fish, as shown in [38] and confirmed in our data. The second activated key player, $C t n n b$, is a member of the Wnt4/ $\beta$-catenin pathway. However, Wnt4 is not over-expressed in sharpsnout seabream female gonads. This pathway is well-studied in mammals (e.g. $[96,97]$ ), but not much is known for teleosts; the few studies on teleosts show that Wnt4 does not follow the pattern observed in mammals [23,40,98]. Finally, two genes that are known markers of ovarian development in mammals tightly linked to Wnt4/ $\beta$-catenin 
pathway, R-spondin (Rspo-1) and Follistatin (Fst), were not differentially expressed in sharpsnout seabream gonads. Rspo-1 is involved in the ovarian differentiation in medaka in early stages of development; in later stages, its expression balances between male and female gonads [99]. Fst has been studied in detail in medaka [23] showing a lack of great differences among gonad types. Given the significant up-regulation of Ctnnb, the Wnt4/ $\beta$ catenin pathway is involved in female gonads function and its significance and role should be deeper studied. Our search for Foxl2, another marker of ovarian development across vertebrates [100], showed that it is expressed similarly in both male and female gonads. This was also the case for the gonads of a protogynous hermaphrodite wrasse, Halichoeres trimaculatus [101], but not for several other gonochoristic fish [e.g. medaka [102,103], tilapia [104], Southern catfish [105], catfish [106], Rare minnow [107], etc.], which suggests that Foxl2 might play distinct roles in the gonads of hermaphrodite fishes. Finally, estrogen receptors did not exhibit any differentiation between the two sexes.

Genes known to have a role in sex determination and differentiation in vertebrates are active in the hermaphrodite sharpsnout seabream, as shown by the expression patterns in the current study. However, it is still open whether the molecular pathways are conserved compared to other teleosts. The current knowledge and comparisons among teleosts show that both upstream and downstream genes alter their position and function in space and time $[23,95]$. Apart from the known candidates, we observed thousands other genes in our data. Those reflect the complex biological processes taking place in the gonads (e.g. cell proliferation, metabolic processes, regulation of transcription, etc.).

\section{Male vs. female brain expression patterns}

Brain has a pronounced sexual dimorphism in function in mammals. However, even in model organisms like human, detailed information is only recently being gathered e.g. [108]. In fish brain, sexual dimorphism is less pronounced in gonochorists compared to mammals, and even less in hermaphrodites [109]. Further, the teleost brain is characterized by remarkable sexual plasticity [110]. Our results showed that in sharpsnout seabream, male and female brains have almost identical expression patterns with few exceptions. The observed divergence was smaller than reported in other fishes (e.g. zebrafish: [25]; rainbow trout: [111]; medaka: [109]). This may be due to reduced sex-specificity in rudimentary hermaphrodites brain.

The discovered genes provide a baseline for understanding the brain sexual divergence in sharpsnout seabream (Figure 4). Like in gonads, more genes are over-expressed in male rather than female brains. These genes are involved in development, metabolism, regulation of transcription, vision, etc. Some are even involved in RNA-dependent DNA replication -probably linked to transposable elements. Notably, none of the known sex determining genes is differentially activated in the brain. The genes over-expressed in male brains include several factors linked to sex in other taxa, such as Tcf12 (involved in estrogen/antiestrogen response in the teleost Fathead minnow [112]), Cbln1 (over-expressed in mouse testis compared to ovaries, as part of the male developmental pathway [113-116]), Rs1 (X-linked gene in human associated to a common macular degeneration in males [117]), Ca12 (involved in the function of uterus in mice [118]), Hyou1 (involved in gonadogenesis in mice [119]) and Aqp1 (possible involvement in the water homeostasis of the male reproductive system [120]). From the genes overexpressed in females, Hamp is regulated by estrogens in mice [113] regulating brain iron metabolism [121]. Other genes that might play significant but still unknown roles in the development of female brain are Alx3, NeoVTX subunit alpha, Thap 9 and Itga2. All those are candidates for regulating and maintaining the sex-specific brain phenotypes and require deeper investigation.

In Sparidae, brain expression has been studied in black porgy; the expression of three Gnrh genes in different developmental stages of ovaries and testis has been characterized and they were found linked to sexual development [38]. However, in sharpsnout seabream all three genes (Gnrh-I-III) have only basal or no expression in both male and female brains. Further, in the gonads we found significant divergence in the expression of aromatase $\alpha$, which had no expression in either male or female brains. On the contrary, we found strong expression of aromatase $\beta$ in the brain and low expression in the gonads of both sexes. In both tissues, no significant expression difference was observed between the sexes for aromatase $\beta$. Those are the expected expression patterns of the two aromatases as observed in other teleosts as well (summarized in [48]). Targeted studies and comparative data from other species will elucidate the role each of those genes play and the mechanisms regulating their expression (e.g. environmental, social, genetic, etc.).

\section{Genetic marker discovery through RNA-sequencing}

The possibility to reconstruct the expressed genic sequences at a global scale through RNA-Seq, gives access to a plethora of genetic markers widely distributed in the genome. We identified a considerable dataset of STR and SNP markers that can be used as raw material in future population genetics, broodstock management, QTL mapping and Marker Assisted Selection (MAS) in sharpsnout seabream and other phylogenetically close sparid species. Discovering SNP markers can be a relatively easy task in a dataset like the one produced here, 
as pooling individuals allows identifying polymorphic sites robustly (although this can be proven only through further targeted experiments). However, further filtering of the SNPs for individual genotyping (see Methods) reduced dramatically the available SNPs. Thus, finding SNP markers that pass certain quality filters for all sequenced individuals is not trivial. To increase the number of informative markers, either deeper sequencing of each individual or use of different genotyping methods would be more appropriate (e.g. RNA-Seq with normalized libraries, Genotyping by Sequencing, SNP-chips scanning, etc.). Apart from SNPs, RNA-Seq leads to the discovery of thousands new STR markers in the transcribed regions of the genome, offering the possibility to select appropriate markers for future analyses.

\section{Conclusions}

In this study, we conducted a comprehensive search of the genes expressed in the male and female gonad and brain tissues of sharpsnout seabream. We used the information extracted from sequencing the RNA of the two tissue types i) to obtain a global view of the sex-specific expression patterns in a rudimentary hermaphrodite teleost and ii) to gather transcriptomic information that will make future comparative analyses feasible. The picture we obtained refers to the particular developmental stage, and averages among all sub-tissues within brain or gonads. In our results, we found the same genes that are responsible for sex differentiation in numerous other species. Some of them might play a role in the sex determination procedure that takes place early in the development. The whole-transcriptome approach employed lays the foundation for future studies to identify genes responsible not only for the sex determination, but also the gonad development and maintenance in sharpsnout seabream. Similar analyses on the tissue parts and of different developmental stages will provide the dynamic view necessary for a complete understanding of sex development.

Eventually, comparison of the expression profiles in sharpsnout seabream with closely related species that display alternative reproductive modes (e.g. the protandrous Sparus aurata and other protogynous species), will offer impressive insights into the particular genetic toolkits deployed in each mechanism.

\section{Availability of supporting data}

Raw reads are deposited in N.C.B.I. sequence read archive under the BioProject ID PRJNA241484.

\section{Additional files}

Additional file 1: Figure S1. Female gonad tissue RNA profile. Total RNA profile of a female gonad as retrieved from an agarose gel and the Agilent Bioanalyzer.
Additional file 2: Figure S2. MA plot for gonad and brain samples. Axes represent $\log _{2}$ fold change versus the mean normalized base counts; significantly differentially expressed loci are shown in red. Genes exceeding the axes range are not shown.

Additional file 3: Table S1. The list of differentially expressed genes in gonad and brain tissues (FDR <0.05).

Additional file 4: Table S2. GO terms over-represented in female or male up-regulated genes in sharpsnout seabream gonads. Fisher's exact test (adjusted p-value $<005$ ) on the GO terms of male versus female gonads over-expressed loci.

Additional file 5: Table S3. Genes associated with sex-related GO terms

Additional file 6: Table S4. Comparative analysis of genes expressed only in the gonads of one sex in sharpsnout seabream.

Additional file 7: Table S5. SNP and STR markers discovered. Additional file 8: Table S6. Individual genotyping for the high quality selected SNP loci.

Additional file 9: Figure S3. PCA conducted on SNP genotypes. The genotypes of each individual from the restricted set of 1009 SNPs are analyzed in a PCA.

Additional file 10: Table S7. Kinship coefficients of the studied individuals.

\section{Competing interests}

The authors declare that they have no competing interests.

\section{Authors' contributions}

TM, AT, ES, CCM, CST designed experiments. CST, CCM, NP conducted sampling. AT performed laboratory experiments. TM, JL analyzed data. JZX performed library construction and sequencing. TM wrote the manuscript with help from other authors. CST conceived and managed the project. All authors read and approved the final version of the manuscript.

\section{Acknowledgements}

Financial support for this study has been provided by the Ministry of Education and Religious Affairs, under the Call "ARISTEIA I" of the National Strategic Reference Framework 2007-2013 (SPARCOMP, \#36), co-funded by the EU and the Hellenic Republic through the European Social Fund. We would like to thank V. Terzoglou and E. Kaitetzidou for help in RNA extractions, Irini Sigelaki for help in sampling and Dr. Hooman Moghadam, Dr. Gianpaolo Zampicinini, Dr. Jon B. Kristoffersen and Ji Hyoun Kang for valuable discussions. Finally, we would like to thank three anonymous reviewers for their valuable comments on the manuscript.

\section{Author details}

${ }^{1}$ Institute of Marine Biology, Biotechnology and Aquaculture (I.M.B.B.C.), Hellenic Centre for Marine Research (H.C.M.R.), Heraklion, Greece. ${ }^{2}$ Genomics Resources Core Facility, Weill Cornell Medical College, New York, USA.

Received: 25 March 2014 Accepted: 30 July 2014

Published: 6 August 2014

\section{References}

1. Bull JJ: Evolution of sex determining mechanisms. Menlo Park, California: Benjamin/Cummings Pub. Co; 1983.

2. Valenzuela N, Adams DC, Janzen FJ: Pattern does not equal process: exactly when is sex environmentally determined? Am Nat 2003, 161:676-683.

3. Ospina-Alvarez N, Piferrer F: Temperature-dependent sex determination in fish revisited: prevalence, a single sex ratio response pattern, and possible effects of climate change. Plos One 2008, 3(7):e2837.

4. Piferrer F: Epigenetics of sex determination and gonadogenesis. Dev Dynam 2013, 242(4):360-370.

5. Sinclair AH, Berta P, Palmer MS, Hawkins JR, Griffiths BL, Smith MJ, Foster JW, Frischauf AM, Lovell-Badge R, Goodfellow PN: A gene from the human sex-determining region encodes a protein with homology to a conserved DNA-binding motif. Nature 1990, 346(6281):240-244. 
6. Koopman P, Gubbay J, Vivian N, Goodfellow P, Lovell-Badge R: Male development of chromosomally female mice transgenic for Sry. Nature 1991, 351(6322):117-121.

7. Smith CA, Roeszler KN, Ohnesorg T, Cummins DM, Farlie PG, Doran TJ, Sinclair AH: The avian Z-linked gene DMRT1 is required for male sex determination in the chicken. Nature 2009, 461(7261):267-271.

8. Yoshimoto S, Okada E, Umemoto H, Tamura K, Uno Y, Nishida-Umehara C, Matsuda Y, Takamatsu N, Shiba T, Ito M: A W-linked DM-domain gene, DM-W, participates in primary ovary development in Xenopus laevis. Proc Natl Acad Sci U S A 2008, 105(7):2469-2474.

9. Matsuda M, Nagahama Y, Shinomiya A, Sato T, Matsuda C, Kobayashi T, Morrey CE, Shibata N, Asakawa S, Shimizu N, Hori H, Hamaguchi S, Sakaizumi M: DMY is a Y-specific DM-domain gene required for male development in the medaka fish. Nature 2002, 417(6888):559-563.

10. Nanda I, Kondo M, Hornung U, Asakawa S, Winkler C, Shimizu A, Shan ZH, Haaf T, Shimizu N, Shima A, Schmid M, Schartl M: A duplicated copy of DMRT1 in the sex-determining region of the $Y$ chromosome of the medaka, Oryzias latipes. Proc Natl Acad Sci U S A 2002, 99(18):11778-11783.

11. Kamiya T, Kai W, Tasumi S, Oka A, Matsunaga T, Mizuno N, Fujita M, Suetake H, Suzuki S, Hosoya S, Tohari S, Brenner S, Miyadai T, Venkatesh B, Suzuki Y, Kikuchi K: A trans-species missense SNP in Amhr2 is associated with sex determination in the Tiger pufferfish, Takifugu rubripes (fugu). Plos Genet 2012, 8(7):e1002798.

12. Yano A, Guyomard R, Nicol B, Jouanno E, Quillet E, Klopp C, Cabau C, Bouchez O, Fostier A, Guiguen Y: An immune-related gene evolved into the master sex-determining gene in Rainbow trout, Oncorhynchus mykiss. Curr Biol 2012, 22(15):1423-1428.

13. Hattori RS, Murai Y, Oura M, Masuda S, Majhi SK, Sakamoto T, Fernandino الJ, Somoza GM, Yokota M, Strussmann CA: A Y-linked anti-Mullerian hormone duplication takes over a critical role in sex determination. Proc Natl Acad Sci U S A 2012, 109(8):2955-2959.

14. Peichel CL, Ross JA, Matson CK, Dickson M, Grimwood J, Schmutz J, Myers RM, Mori S, Schluter D, Kingsley DM: The master sex-determination locus in threespine sticklebacks is on a nascent $Y$ chromosome. Curr Biol 2004, 14(16):1416-1424.

15. Palaiokostas C, Bekaert M, Khan MGQ, Taggart JB, Gharbi K, McAndrew BJ, Penman DJ: Mapping and validation of the major sex-determining region in Nile tilapia (Oreochromis niloticus L.) using RAD sequencing. Plos One 2013, 8(7):e68389.

16. Palaiokostas C, Bekaert M, Davie A, Cowan ME, Oral M, Taggart JB, Gharbi K, McAndrew BJ, Penman DJ, Migaud H: Mapping the sex determination locus in the Atlantic halibut (Hippoglossus hippoglossus) using RAD sequencing. BMC Genomics 2013, 14:566.

17. Martinez P, Bouza C, Hermida M, Fernandez J, Toro MA, Vera M, Pardo B, Millan A, Fernandez C, Vilas R, Sánchez L, Felip A, Piferrer F, Ferreiro I, Cabaleiro $S$ : Identification of the major sex-determining region of turbot (Scophthalmus maximus). Genetics 2009, 183(4):1443-1452.

18. Bradley KM, Breyer JP, Melville DB, Broman KW, Knapik EW, Smith JR: An SNP-based linkage map for zebrafish reveals sex determination loci. G3 2011, 1(1):3-9.

19. Loukovitis D, Sarropoulou E, Batargias C, Apostolidis AP, Kotoulas G, Tsigenopoulos CS, Chatziplis D: Quantitative trait loci for body growth and sex determination in the hermaphrodite teleost fish Sparus aurata $L$. Anim Genet 2012, 43(6):753-759.

20. Loukovitis D, Sarropoulou E, Tsigenopoulos CS, Batargias C, Magoulas A, Apostolidis AP, Chatziplis D, Kotoulas G: Quantitative trait loci involved in sex determination and body growth in the Gilthead sea bream (Sparus aurata L.) through targeted genome scan. Plos One 2011, 6(1):e16599.

21. Marín I, Baker BS: The evolutionary dynamics of sex determination. Science 1998, 281(5385):1990-1994.

22. Graham P, Penn JK, Schedl P: Masters change, slaves remain. BioEssays 2003, 25(1):1-4.

23. Herpin A, Adolfi MC, Nicol B, Hinzmann M, Schmidt C, Klughammer J, Engel M, Tanaka M, Guiguen Y, Schartl M: Divergent expression regulation of gonad development genes in medaka shows incomplete conservation of the downstream regulatory network of vertebrate sex determination. Mol Biol Evol 2013, 30(10):2328-2346

24. Piferrer F, Ribas L, Diaz N: Genomic approaches to study genetic and environmental influences on fish sex determination and differentiation. Mar Biotechnol 2012, 14(5):591-604
25. Sreenivasan R, Cai MN, Bartfai R, Wang XG, Christoffels A, Orban L: Transcriptomic analyses reveal novel genes with sexually dimorphic expression in the zebrafish gonad and brain. Plos One 2008, 3(3):e1791

26. Small CM, Carney GE, Mo QX, Vannucci M, Jones AG: A microarray analysis of sex- and gonad-biased gene expression in the zebrafish: evidence for masculinization of the transcriptome. BMC Genomics 2009, 10:579.

27. Zhang ZP, Wang YL, Wang SH, Liu JT, Warren W, Mitreva M, Walter RB: Transcriptome analysis of female and male Xiphophorus maculatus Jp 163 A. Plos One 2011, 6(4):e18379.

28. Forconi M, Canapa A, Barucca M, Biscotti MA, Capriglione T, Buonocore F, Fausto AM, Makapedua DM, Pallavicini A, Gerdol M, De Moro G, Scapigliati G, Olmo E, Schartl M: Characterization of sex determination and sex differentiation genes in Latimeria. Plos One 2013, 8(4):e56006.

29. Sun FY, Liu SK, Gao XY, Jiang YL, Perera D, Wang $X L$, Li C, Sun LY, Zhang JR, Kaltenboeck L, Dunham R, Liu Z: Male-biased genes in catfish as revealed by RNA-Seq analysis of the testis transcriptome. Plos One 2013, 8(7):e68452.

30. Tao WJ, Yuan J, Zhou LY, Sun LN, Sun YL, Yang SJ, Li MH, Zeng S, Huang BF, Wang $\mathrm{DH}$ : Characterization of gonadal transcriptomes from Nile tilapia (Oreochromis niloticus) reveals differentially expressed genes. Plos One 2013, 8(5):e63604.

31. Erisman BE, Petersen CW, Hastings PA, Warner RR: Phylogenetic perspectives on the evolution of functional hermaphroditism in teleost fishes. Integr Comp Biol 2013, 53(4):736-754.

32. Buxton $C D$, Garratt PA: Alternative reproductive styles in seabreams (Pisces, Sparidae). Environ Biol Fish 1990, 28(1-4):113-124.

33. Atz JW: Intersexuality in Fishes. In Intersexuality in Vertebrates Including Man. Edited by Marshall AJ, Armstrong CN. New York: Academic Press; 1964:145-232.

34. Mylonas CC, Zohar Y, Pankhurst N, Kagawa H: Reproduction and broodstock management. In Sparidae. Edited by Pavlidis MA, Mylonas CC. Oxford, UK: Wiley-Blackwell; 2011:95-131.

35. Wu GC, Chang CF: The switch of secondary sex determination in protandrous black porgy, Acanthopagrus schlegeli. Fish Physiol Biochem 2013, 39(1):33-38.

36. He CL, Du JL, Lee YH, Sun LT, Chang CF: Differential Dmrt1 transcripts in gonads of the protandrous black porgy, Acanthopagrus schlegeli. Cytogenet Genome Res 2003, 101:309-313.

37. Wu GC, Tomy S, Chang CF: The expression of nr0b1 and nr5a4 during gonad development and sex change in protandrous black porgy fish, Acanthopagrus schlegeli. Biol Reprod 2008, 78(2):200-210.

38. Wu GC, Tomy S, Lee MF, Lee YH, Yueh WS, Lin CJ, Lau EL, Chang CF: Sex differentiation and sex change in the protandrous black porgy, Acanthopagrus schlegeli. Gen Comp Endocrinol 2010, 167(3):417-421.

39. Wu GC, Tomy S, Nakamura M, Chang CF: Dual Roles of cyp19a1a in gonadal sex differentiation and development in the protandrous black porgy, Acanthopagrus schlegeli. Biol Reprod 2008, 79(6):1111-1120.

40. Wu GC, Chang CF: wnt4 as associated with the development of ovarian tissue in the protandrous black porgy, Acanthopagrus schlegeli. Biol Reprod 2009, 81(6):1073-1082.

41. Tomy S, Wu GC, Huang HR, Chang CF: Age-dependent differential expression of genes involved in steroid signalling pathway in the brain of protandrous black porgy, Acanthopagrus schlegeli. Dev Neurobiol 2009, 69(5):299-313.

42. Tomy S, Wu GC, Huang HR, Dufour S, Chang CF: Developmental expression of key steroidogenic enzymes in the brain of protandrous black porgy fish, Acanthopagrus schlegeli. J Neuroendocrinol 2007, 19(8):643-655.

43. Micale V, Perdichizzi F, Basciano G: Aspects of the reproductive biology of the sharpsnout seabream Diplodus puntazzo (Cetti, 1777).1. Gametogenesis and gonadal cycle in captivity during the third year of life. Aquaculture 1996, 140(3):281-291

44. Pajuelo JG, Lorenzo JM, Dominguez-Seoane R: Gonadal development and spawning cycle in the digynic hermaphrodite sharpsnout seabream Diplodus puntazzo (Sparidae) off the Canary Islands, northwest of Africa. J Appl Ichthyol 2008, 24(1):68-76.

45. Klimogianni A, Kalanji M, Pyrenis G, Zoulioti A, Trakos G: Ontogeny of embryonic and yolk-sac larval stage of the sparid sharpsnout sea bream (Diplodus puntazzo Cetti, 1777). J Fish Aquat Sci 2011, 6:62-73.

46. Papadaki M, Papadopoulou M, Siggelaki I, Mylonas CC: Egg and sperm production and quality of sharpsnout sea bream (Diplodus puntazzo) in captivity. Aquaculture 2008, 276(1-4):187-197. 
47. Wang Z, Gerstein M, Snyder M: RNA-Seq: a revolutionary tool for transcriptomics. Nat Rev Genet 2009, 10(1):57-63.

48. Le Page Y, Diotel N, Vaillant C, Pellegrini E, Anglade I, Merot Y, Kah O: Aromatase, brain sexualization and plasticity: the fish paradigm. Eur $\mathrm{J}$ Neurosci 2010, 32(12):2105-2115.

49. Guidelines for the treatment of animals in behavioural research and teaching. Anim Behav 2001, 61(1):271-275. http://www.ncbi.nlm.nih.gov/ pubmed/11170716.

50. de Diaz Cerio O, Rojo-Bartolome I, Bizarro C, Ortiz-Zarragoitia M, Cancio I: $5 \mathrm{~S}$ rRNA and accompanying proteins in gonads: powerful markers to identify sex and reproductive endocrine disruption in fish. Environ sci Tech 2012, 46(14):7763-7771.

51. Babraham Bioinformatics: FastQC A quality control tool for high throughput sequence data. http://www.bioinformatics.babraham.ac.uk/projects/fastqc/.

52. FASTX-Toolkit software package: http://hannonlab.cshl.edu/fastx_toolkit/.

53. Luo R, Liu B, Xie Y, Li Z, Huang W, Yuan J, He G, Chen Y, Pan Q, Liu Y, Tang J, Wu G, Zhang H, Shi Y, Liu Y, Yu C, Wang B, Lu Y, Han C, Cheung DW, Yiu SM, Peng S, Xiaoqian Z, Liu G, Liao X, Li Y, Yang H, Wang J, Lam TW, Wang J: SOAPdenovo2: an empirically improved memory-efficient short-read de novo assembler. GigaScience 2012, 1(1):18.

54. Zerbino DR, Birney E: Velvet: algorithms for de novo short read assembly using de Bruijn graphs. Genome Res 2008, 18(5):821-829.

55. Schulz $M H$, Zerbino DR, Vingron M, Birney E: Oases: robust de novo RNA-seq assembly across the dynamic range of expression levels. Bioinformatics 2012, 28(8):1086-1092.

56. Grabherr MG, Haas BJ, Yassour M, Levin JZ, Thompson DA, Amit I, Adiconis X, Fan L, Raychowdhury R, Zeng QD, Chen Z, Mauceli E, Hacohen N, Gnirke A, Rhind N, di Palma F, Birren BW, Nusbaum C, Lindblad-Toh K, Friedman N, Regev A: Full-length transcriptome assembly from RNA-Seq data without a reference genome. Nat Biotechnol 2011, 29(7):644-U130.

57. Altschul SF, Gish W, Miller W, Myers EW, Lipman DJ: Basic local alignment search tool. J Mol Biol 1990, 215(3):403-410.

58. Flicek P, Ahmed I, Amode MR, Barrell D, Beal K, Brent S, Carvalho-Silva D, Clapham P, Coates G, Fairley S, Fitzgerald S, Gil L, García-Girón C, Gordon L, Hourlier T, Hunt S, Juettemann T, Kähäri AK, Keenan S, Komorowska M, Kulesha E, Longden I, Maurel T, McLaren WM, Muffato M, Nag R, Overduin B, Pignatelli M, Pritchard B, Pritchard E, et al: Ensembl 2013. Nucleic Acids Res 2013, 41(D1):D48-D55.

59. Langmead B, Trapnell C, Pop M, Salzberg SL: Ultrafast and memoryefficient alignment of short DNA sequences to the human genome. Genome Biol 2009, 10(3):R25.

60. Li B, Dewey CN: RSEM: accurate transcript quantification from RNA-Seq data with or without a reference genome. BMC Bioinformatics 2011, 12:323.

61. Haas BJ, Papanicolaou A, Yassour M, Grabherr M, Blood PD, Bowden J, Couger MB, Eccles D, Li B, Lieber M, Macmanes MD, Ott M, Orvis J, Pochet N, Strozzi F, Weeks N, Westerman R, William T, Dewey CN, Henschel R, Leduc RD, Friedman $N$, Regev $A$ : De novo transcript sequence reconstruction from RNA-seq using the trinity platform for reference generation and analysis. Nat Protoc 2013, 8(8):1494-1512.

62. Lagnel J, Tsigenopoulos CS, lliopoulos I: NOBLAST and JAMBLAST: new options for BLAST and a java application manager for BLAST results. Bioinformatics 2009, 25(6):824-826.

63. Conesa A, Gotz S, Garcia-Gomez JM, Terol J, Talon M, Robles M: Blast2GO: a universal tool for annotation, visualization and analysis in functional genomics research. Bioinformatics 2005, 21(18):3674-3676.

64. Zdobnov EM, Apweiler R: InterProScan - an integration platform for the signature-recognition methods in InterPro. Bioinformatics 2001, 17(9):847-848

65. Anders S, Huber W: Differential expression analysis for sequence count data. Genome Biol 2010, 11(10):R106.

66. Rapaport F, Khanin R, Liang Y, Pirun M, Krek A, Zumbo P, Mason CE, Socci ND, Betel $D$ : Comprehensive evaluation of differential gene expression analysis methods for RNA-seq data. Genome Biol 2013, 14(9):R95.

67. Soneson C, Delorenzi M: A comparison of methods for differential expression analysis of RNA-seq data. BMC Bioinformatics 2013, 14:91.

68. Mayer C: Phobos: a tandem repeat search tool. http://www.ruhr-uni-bochum. de/ecoevo/cm/cm_phobos.htm.

69. Li H, Handsaker B, Wysoker A, Fennell T, Ruan J, Homer N, Marth G, Abecasis G, Durbin R, Genome Project Data Processing S: The sequence alignment/map format and SAMtools. Bioinformatics 2009, 25(16):2078-2079.
70. Danecek P, Auton A, Abecasis G, Albers CA, Banks E, DePristo MA Handsaker RE, Lunter G, Marth GT, Sherry ST, McVean G, Durbin R: The variant call format and VCFtools. Bioinformatics 2011, 27(15):2156-2158.

71. Barnett DW, Garrison EK, Quinlan AR, Stromberg MP, Marth GT: BamTools: a $\mathrm{C}++\mathrm{API}$ and toolkit for analyzing and managing BAM files. Bioinformatics 2011, 27(12):1691-1692.

72. Quinn EM, Cormican P, Kenny EM, Hill M, Anney R, Gill M, Corvin AP, Morris DW: Development of strategies for SNP detection in RNA-seq data: application to lymphoblastoid cell lines and evaluation using 1000 Genomes data. Plos One 2013, 8(3):e58815.

73. Zheng X, Levine D, Shen J, Gogarten SM, Laurie C, Weir BS: A highperformance computing toolset for relatedness and principal component analysis of SNP data. Bioinformatics 2012, 28(24):3326-3328.

74. Zarkower D: DMRT Genes in vertebrate gametogenesis. Curr Top Dev Biol 2013, 102:327-356

75. Matson CK, Zarkower D: Sex and the singular DM domain: insights into sexual regulation, evolution and plasticity. Nat Rev Genet 2012, 13(3):163-174

76. Herpin A, Schartl M: Dmrt1 genes at the crossroads: a widespread and central class of sexual development factors in fish. Febs J 2011, 278(7):1010-1019.

77. Wu GC, Chiu PC, Lin CJ, Lyu YS, Lan DS, Chang CF: Testicular dmrt1 Is involved in the sexual fate of the ovotestis in the protandrous black porgy. Biol Reprod 2012, 86(2):41.

78. Wu GC, Chiu PC, Lyu YS, Chang CF: The expression of amh and amhr2 is associated with the development of gonadal tissue and sex change in the protandrous black porgy, Acanthopagrus schlegeli. Biol Reprod 2010, 83(3):443-453.

79. Sekido R, Lovell-Badge R: Sex determination involves synergistic action of SRY and SF1 on a specific Sox9 enhancer. Nature 2008, 453(7197):930-934.

80. Berbejillo J, Martinez-Bengochea A, Bedo G, Brunet F, Volff JN, VizzianoCantonnet D: Expression and phylogeny of candidate genes for sex differentiation in a primitive fish species, the Siberian sturgeon, Acipenser baerii. Mol Reprod Dev 2012, 79(8):504-516.

81. Smith EK, Guzman JM, Luckenbach JA: Molecular cloning, characterization, and sexually dimorphic expression of five major sex differentiationrelated genes in a Scorpaeniform fish, sablefish (Anoplopoma fimbria). Comp Biochem Phys B 2013, 165(2):125-137.

82. Kong Y, Grimaldi M, Curtin E, Dougherty M, Kaufman C, White RM, Zon LI, Liao EC: Neural crest development and craniofacial morphogenesis is coordinated by nitric oxide and histone acetylation. Chem Biol 2014, 21(4):488-501.

83. Wang DS, Kobayashi T, Senthilkumaran B, Sakai F, Sudhakumari CC, Suzuki T, Yoshikuni M, Matsuda M, Morohashi K, Nagahama Y: Molecular cloning of DAX1 and SHP cDNAs and their expression patterns in the Nile tilapia, Oreochromis niloticus. Biochem Bioph Res Co 2002, 297(3):632-640.

84. Martins RS, Deloffre LA, Mylonas CC, Power DM, Canario AV: Developmental expression of DAX1 in the European sea bass, Dicentrarchus labrax: lack of evidence for sexual dimorphism during sex differentiation. Reprod Biol Endocrinol 2007, 5:19.

85. Nakamoto M, Wang DS, Suzuki A, Matsuda M, Nagahama Y, Shibata N: Dax1 suppresses P450arom expression in medaka ovarian follicles. Mol Reprod Dev 2007, 74(10):1239-1246.

86. Bitgood MJ, Shen LY, McMahon AP: Sertoli cell signaling by Desert hedgehog regulates the male germline. Curr Biol 1996, 6(3):298-304.

87. Clark AM, Garland KK, Russell LD: Desert hedgehog (Dhh) gene is required in the mouse testis for formation of adult-type Leydig cells and normal development of peritubular cells and seminiferous tubules. Biol Reprod 2000, 63(6):1825-1838

88. O'Hara WA, Azar WJ, Behringer RR, Renfree MB, Pask AJ: Desert hedgehog is a mammal-specific gene expressed during testicular and ovarian development in a marsupial. BMC Dev Biol 2011, 11:72.

89. Spicer LJ, Sudo S, Aad PY, Wang LS, Chun SY, Ben-Shlomo I, Klein C, Hsueh AJW: The hedgehog-patched signaling pathway and function in the mammalian ovary: a novel role for hedgehog proteins in stimulating proliferation and steroidogenesis of theca cells. Reproduction 2009, 138(2):329-339.

90. Wijgerde M, Ooms M, Hoogerbrugge JW, Grootegoed JA: Hedgehog signaling in mouse ovary: Indian hedgehog and desert hedgehog from granulosa cells induce target gene expression in developing theca cells. Endocrinology 2005, 146(8):3558-3566 
91. Guiguen Y, Fostier A, Piferrer F, Chang CF: Ovarian aromatase and estrogens: a pivotal role for gonadal sex differentiation and sex change in fish. Gen Comp Endocrinol 2010, 165(3):352-366.

92. Kitano T, Takamune K, Kobayashi T, Nagahama Y, Abe SI: Suppression of P450 aromatase gene expression in sex-reversed males produced by rearing genetically female larvae at a high water temperature during a period of sex differentiation in the Japanese flounder (Paralichthys olivaceus). J Mol Endocrinol 1999, 23(2):167-176.

93. Uchida D, Yamashita M, Kitano T, Iguchi T: An aromatase inhibitor or high water temperature induce oocyte apoptosis and depletion of P450 aromatase activity in the gonads of genetic female zebrafish during sex-reversal. Comp Biochem Physiol Mol Integr Physiol 2004, 137(1):11-20.

94. Karube M, Fernandino Jl, Strobl-Mazzulla P, Strussmann CA, Yoshizaki G, Somoza GM, Patino R: Characterization and expression profile of the ovarian cytochrome $\mathrm{p}-450$ aromatase (cyp19A1) gene during thermolabile sex determination in pejerrey, Odontesthes bonariensis. J Exp Zool Part A 2007, 307A(11):625-636

95. Bohne A, Heule C, Boileau N, Salzburger W: Expression and sequence evolution of aromatase cyp19a1 and other sexual development genes in East African cichlid fishes. Mol Biol Evol 2013, 30(10):2268-2285.

96. Chassot AA, Bradford ST, Auguste A, Gregoire EP, Pailhoux E, de Rooij DG, Schedl A, Chaboissier MC: WNT4 and RSPO1 together are required for cell proliferation in the early mouse gonad. Development 2012, 139(23):4461-4472.

97. Li L, Ji SY, Yang JL, Li XX, Zhang J, Zhang Y, Hu ZY, Liu YX: Wnt/betacatenin signaling regulates follicular development by modulating the expression of Foxo3a signaling components. Mol Cell Endocrinol 2013, 382(2):915-925.

98. Nicol B, Guerin A, Fostier A, Guiguen Y: Ovary-predominant wnt4 expression during gonadal differentiation is not conserved in the rainbow trout (Oncorhynchus mykiss). Mol Reprod Dev 2012, 79(1):51-63.

99. Zhou LY, Charkraborty T, Yu XG, Wu LM, Liu G, Mohapatra S, Wang DS, Nagahama $Y$ : R-spondins are involved in the ovarian differentiation in a teleost, medaka (Oryzias latipes). BMC Dev Biol 2012, 12:36.

100. Benayoun BA, Dipietromaria A, Bazin C, Veitia RA: FOXL2: At the crossroads of female sex determination and ovarian function. Adv Exp Med Biol 2009, 665:207-226.

101. Kobayashi $Y$, Horiguchi R, Nozu R, Nakamura M: Expression and localization of forkhead transcriptional factor 2 (Foxl2) in the gonads of protogynous wrasse, Halichoeres trimaculatus. Biol Sex Differ 2010, 1(1):3.

102. Nakamoto M, Matsuda M, Wang DS, Nagahama Y, Shibata N: Molecular cloning and analysis of gonadal expression of Foxl2 in the medaka, Oryzias latipes. Biochem Bioph Res Co 2006, 344(1):353-361.

103. Nakamoto M, Muramatsu S, Yoshida S, Matsuda M, Nagahama Y, Shibatal N: Gonadal sex differentiation and expression of Sox9a2, Dmrt1, and Foxl2 in Oryzias luzonensis. Genesis 2009, 47(5):289-299.

104. Wang DS, Kobayashi T, Zhou LY, Nagahama Y: Molecular cloning and gene expression of Foxl2 in the Nile tilapia, Oreochromis niloticus. Biochem Bioph Res Co 2004, 320(1):83-89.

105. Liu Z, Wu F, Jiao B, Zhang X, Hu C, Huang B, Zhou L, Huang X, Wang Z, Zhang Y, Nagahama Y, Cheng CH, Wang D: Molecular cloning of doublesex and mab-3-related transcription factor 1, forkhead transcription factor gene 2, and two types of cytochrome P450 aromatase in Southern catfish and their possible roles in sex differentiation. J Endocrinol 2007, 194(1):223-241.

106. Sridevi P, Senthilkumaran B: Cloning and differential expression of FOXL2 during ovarian development and recrudescence of the catfish, Clarias gariepinus. Gen Comp Endocrinol 2011, 174(3):259-268.

107. Jiang W, Yang Y, Zhao D, Liu X, Duan J, Xie S, Zhao H: Effects of sexual steroids on the expression of foxl2 in Gobiocypris rarus. Comp Biochem Physiol B: Biochem Mol Biol 2011, 160(4):187-193.

108. Trabzuni D, Ramasamy A, Imran S, Walker R, Smith C, Weale ME, Hardy J, Ryten M, Expression NAB: Widespread sex differences in gene expression and splicing in the adult human brain. Nat Commun 2013, 4:2771.

109. Hiraki T, Takeuchi A, Tsumaki T, Zempo B, Kanda S, Oka Y, Nagahama Y, Okubo K: Female-specific target sites for both oestrogen and androgen in the teleost brain. Proc Bio/ Sci 2012, 279(1749):5014-5023.

110. Godwin J: Neuroendocrinology of sexual plasticity in teleost fishes. Front Neuroendocrinol 2010, 31(2):203-216.

111. Vizziano-Cantonnet D, Anglade I, Pellegrini E, Gueguen MM, Fostier A, Guiguen $Y$, Kah O: Sexual dimorphism in the brain aromatase expression and activity, and in the central expression of other steroidogenic enzymes during the period of sex differentiation in monosex rainbow trout populations. Gen Comp Endocrinol 2011, 170(2):346-355.

112. Garcia-Reyero N, Kroll KJ, Liu L, Orlando EF, Watanabe KH, Sepulveda MS, Villeneuve DL, Perkins EJ, Ankley GT, Denslow ND: Gene expression responses in male fathead minnows exposed to binary mixtures of an estrogen and antiestrogen. BMC Genomics 2009, 10:308.

113. Ikeda Y, Tajima S, Izawa-Ishizawa Y, Kihira Y, Ishizawa K, Tomita S, Tsuchiya K, Tamaki T: Estrogen regulates hepcidin expression via GPR30-BMP6dependent signaling in hepatocytes. Plos One 2012, 7(7):115f.

114. Nef S, Schaad O, Stallings NR, Cederroth CR, Pitetti JL, Schaer G, Malki S, Dubois-Dauphin M, Boizet-Bonhoure B, Descombes P, Parker KL, Vassalli JD: Gene expression during sex determination reveals a robust female genetic program at the onset of ovarian development. Dev Biol 2005, 287(2):361-377.

115. Coveney D, Ross AJ, Slone JD, Capel B: A microarray analysis of the XX Wnt4 mutant gonad targeted at the identification of genes involved in testis vascular differentiation. Gene Expr Patterns 2007, 7(1-2):82-92.

116. Menke DB, Page DC: Sexually dimorphic gene expression in the developing mouse gonad. Gene Expr Patterns 2002, 2(3-4):359-367.

117. Friedrich U, Stohr $H$, Hilfinger D, Loenhardt T, Schachner M, Langmann T, Weber BH: The Na/K-ATPase is obligatory for membrane anchorage of retinoschisin, the protein involved in the pathogenesis of X-linked juvenile retinoschisis. Hum Mol Genet 2011, 20(6):1132-1142.

118. Gholami K, Muniandy S, Salleh N: In-vivo functional study on the involvement of CFTR, SLC26A6, NHE-1 and CA isoenzymes II and XII in uterine fluid $\mathrm{pH}$, volume and electrolyte regulation in rats under different sex-steroid influence. Int J Med Sci 2013, 10(9):1121-1134.

119. Ewen K, Baker M, Wilhelm D, Aitken RJ, Koopman P: Global survey of protein expression during gonadal sex determination in mice. Mol Cell Proteomics 2009, 8(12):2624-2641.

120. Skowronski MT, Leska A, Robak A, Nielsen S: Immunolocalization of aquaporin-1, -5 , and -7 in the avian testis and vas deferens. $J$ Histochem Cytochem 2009, 57(10):915-922.

121. Wang SM, Fu LJ, Duan XL, Crooks DR, Yu P, Qian ZM, Di XJ, Li J, Rouault TA, Chang YZ: Role of hepcidin in murine brain iron metabolism. Cell Mol Life Sci 2010, 67(1):123-133.

doi:10.1186/1471-2164-15-655

Cite this article as: Manousaki et al:: The sex-specific transcriptome of the hermaphrodite sparid sharpsnout seabream (Diplodus puntazzo). BMC Genomics 2014 15:655.

\section{Submit your next manuscript to BioMed Central and take full advantage of:}

- Convenient online submission

- Thorough peer review

- No space constraints or color figure charges

- Immediate publication on acceptance

- Inclusion in PubMed, CAS, Scopus and Google Scholar

- Research which is freely available for redistribution

Submit your manuscript at www.biomedcentral.com/submit
C Biomed Central 